\title{
Evaluation of Institute of Medicine Guidelines for Gestational Weight Gain in Women with Chronic Hypertension
}

\author{
Anne M. Siegel, MD ${ }^{1}$ Alan T. Tita, MD, PhD ${ }^{1} \quad$ Hannah Machemehl, MD ${ }^{1} \quad$ Joseph R. Biggio, MD ${ }^{1}$ \\ Lorie M. Harper, MD, $\mathrm{MSCl}^{1}$ \\ ${ }^{1}$ Division of Maternal Fetal Medicine, Department of Obstetrics and
Gynecology, Center for Women's Reproductive Health at the
University of Alabama at Birmingham, Birmingham, Alabama \\ Am J Perinatol Rep 2017;7:e145-e150. \\ Address for correspondence Anne M. Siegel, MD, Department of \\ Obstetrics and Gynecology, The University of Alabama at \\ Birmingham, 1700 6th Ave, South Ste 10270, Birmingham, AL 35233 \\ (e-mail: asiege4@gmail.com).
}

\begin{abstract}
Keywords

- hypertension

- gestational weight gain

- preeclampsia

- pregnancy

Objective To assess the impact of gestational weight gain (GWG) outside the Institute of Medicine (IOM) recommendations on perinatal outcomes in pregnancies complicated by chronic hypertension (HTN).

Methods The study consisted of a retrospective cohort of all singletons with HTN from 2000 to 2014. Maternal outcomes examined were superimposed preeclampsia and cesarean delivery. Neonatal outcomes were small for gestational age (SGA), large for gestational age (LGA), and preterm birth (PTB). Groups were compared using analysis of variance and chi-squared test for trend. Backward stepwise logistic regression was adjusted for confounding factors.

Results Of 702 subjects, $106(15.1 \%)$ gained within, 176 (25.0\%) gained less, and 420 $(59.8 \%)$ gained more weight than the IOM recommendations. After adjusting for confounders, GWG above IOM recommendations remained associated with LGA (adjusted odds ratio [AOR]: 2.53, confidence interval [CI] 95\%:1.29-4.95). Weight gain less than recommended was associated with a decreased risk of superimposed preeclampsia (AOR: $0.49, \mathrm{Cl} 95 \%$ : 0.26-0.93) without increasing the risk of SGA (AOR: 1.03, Cl 95\%: 0.57-1.86).

Conclusion Women with pregnancies complicated by chronic HTN should be counseled regarding the association of LGA with excessive GWG. Additionally, they should be counseled that weight gain below recommendations may be associated with a decreased risk of superimposed preeclampsia; however, this association deserves further investigation.
\end{abstract}

Chronic hypertension (HTN) complicates approximately 3\% of pregnancies in the United States ${ }^{1}$ and increases the risk of poor maternal and neonatal outcomes, including superimposed preeclampsia, placental abruption, cesarean delivery, stroke, renal or cardiac failure, miscarriage, stillbirth, growth restriction, prematurity, and neonatal death.

received

March 7, 2017

accepted after revision

May 25, 2017
DOI https://doi.org/

10.1055/s-0037-1604076. ISSN 2157-6998.
The Institute of Medicine (IOM) has developed guidelines for gestational weight gain (GWG) to target an ideal birth weight. ${ }^{2}$ However, these guidelines were developed in a healthy population, and no specific guidelines were created for special populations such as women with chronic (HTN). Because women with chronic HTN are at increased risk of
Copyright $\odot 2017$ by Thieme Medical Publishers, Inc., 333 Seventh Avenue, New York, NY 10001, USA. Tel: +1(212) 584-4662.
License terms

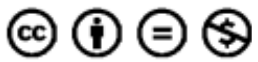


fetal growth restriction due to placental insufficiency, ${ }^{3,4}$ low maternal weight gain may adversely impact women with chronic HTN, and weight gain above the guidelines may be advantageous in achieving an ideal birth weight. Alternatively, excessive weight gain in both normal weight and obese populations is associated with an increased risk of pregnancy-related hypertension. ${ }^{5,6}$ Given these conflicting associations, GWG in this population may be of increased significance.

Superimposed preeclampsia is a common complication of chronic hypertension. Preeclampsia frequently leads to preterm birth, neonatal morbidity, and perinatal death. Additionally, excessive GWG in low-risk pregnancies also impact the risk of preeclampsia and cesarean delivery. ${ }^{7}$ Therefore, in pregnancies complicated by chronic hypertension, the association of GWG with hypertensive disorders of pregnancy, cesarean delivery, and subsequent neonatal outcomes are of particular interest.

Therefore, we aimed to assess the impact of GWG outside the IOM recommendations on perinatal outcomes in pregnancies complicated by chronic HTN. We hypothesized that weight gain above the IOM recommendations is associated with an increased risk of LGA, superimposed preeclampsia, cesarean delivery, and preterm birth.

\section{Materials and Methods}

This was a retrospective cohort study of all singleton pregnancies at a tertiary care center complicated by chronic HTN between 2000 and 2014. The study period was determined by the availability of the complete medical record for review. Institutional review board (IRB) approval was obtained from the University of Alabama at Birmingham.

Subjects were identified by a diagnosis of chronic HTN in our searchable electronic medical records. Trained chart abstractors completed standardized chart abstraction forms from the medical record, confirming the diagnosis of chronic HTN by blood pressure criteria (blood pressures $\geq 140$ / $90 \mathrm{~mm} \mathrm{Hg}$ prior to pregnancy or prior to 20 weeks gestation and/or medication history including the use of antihypertensive medications prior to pregnancy). Data were collected on maternal demographics, medical and obstetrical history, medication use, prenatal blood pressures, labor and delivery events, and neonatal outcomes. Chart abstractors recorded blood pressures, weight, and urine dipstick information from each prenatal visit. All women were treated under the supervision of Maternal-Fetal Medicine specialists. As per the institutional protocol, antihypertensive medications were initiated or adjusted to achieve blood pressures $\leq 150 / 90$. Women were evaluated for superimposed preeclampsia if blood pressures were acutely or persistently elevated to $\geq 140 / 90 \mathrm{~mm} \mathrm{Hg}$ after 20 weeks gestation. Women were excluded for incomplete body mass index (BMI) data, no documented weight within 2 weeks of delivery, fetal anomalies, repeat pregnancies within the study period, and major maternal comorbidity other than chronic hypertension, renal disease, or diabetes. We elected to include gestational diabetes, pregestational diabetes, and baseline renal disease (defined as creatinine $\geq 1.2 \mathrm{mg} / \mathrm{dL}$ or $\geq 300 \mathrm{mg} /$ day of proteinuria at $<20$ weeks) in the analysis, as these are frequent comorbidities.

The IOM makes recommendations for GWG per week of pregnancy in the second and third trimesters assuming a 0.5 to $2 \mathrm{~kg}$ weight gain in the first trimester. ${ }^{2}$ We calculated the weekly GWG in the second and third trimesters for the cohort in the following way. First, we identified the first documented weight between 14 and 20 weeks. The final measured weight (within 2 weeks of delivery) was used to calculate the total weight gain for the second and third trimesters. A recorded GWG per week was calculated by dividing the total GWG by the number of weeks between delivery and the first measured weight. As prepregnancy weight was frequently missing or self-reported, this strategy avoided using the IOM presumed 0.5 to $2 \mathrm{~kg}$ first trimester weight gain and defined the exposure with a measured second and third trimester weight gain.

Women were classified as GWG within, less than, or greater than IOM recommendations for BMI at the first measured weight. Per week of pregnancy in the second and third trimesters, the IOM recommends underweight women (BMI $<18.5 \mathrm{~kg} / \mathrm{m}^{2}$ ) to gain 0.44 to $0.58 \mathrm{~kg} /$ week, normal weight women (BMI: $18.5-24.9 \mathrm{~kg} / \mathrm{m}^{2}$ ) to gain 0.35 to $0.50 \mathrm{~kg} /$ week, overweight women (BMI: $25.0-29.9 \mathrm{~kg} / \mathrm{m}^{2}$ ) to gain 0.23 to $0.33 \mathrm{~kg} /$ week, and obese women (BMI $\geq 30 \mathrm{~kg} /$ $\mathrm{m}^{2}$ ) to gain 0.17 to $0.27 \mathrm{~kg} /$ week. $^{2}$

The primary maternal outcome examined was superimposed preeclampsia; the secondary maternal outcomes were superimposed preeclampsia with severe features and cesarean delivery. Superimposed preeclampsia was determined by a review of the medical record demonstrating elevated blood pressures and laboratory values consistent with the American College of Obstetricians and Gynecologists (ACOG) definition of preeclampsia based on ACOG Task Force Document from $2013 .{ }^{8}$ Superimposed preeclampsia was defined as blood pressures $\geq 140 / 90 \mathrm{~mm} \mathrm{Hg}$ with evidence of proteinuria ( $\geq 0.3$ urine protein/creatinine ratio or $\geq 300 \mathrm{mg} / 24$ hours) or a serum laboratory abnormality. Hypertension alone was not sufficient for a diagnosis of superimposed preeclampsia. Superimposed preeclampsia with severe features was defined as blood pressures $\geq 140 / 90 \mathrm{~mm} \mathrm{Hg}$ with at least one of the following features: blood pressures $\geq 160$ / $110 \mathrm{~mm} \mathrm{Hg}$ in the presence of proteinuria, thrombocytopenia $(<100,000$ platelets $/ \mathrm{mL})$, creatinine $\geq 1.2 \mathrm{mg} / \mathrm{dL}$, and aspartate transaminase (AST) $>80 \mathrm{IU} / \mathrm{mL}$ (twice the upper range of normal at our institution). Neurologic symptoms were not abstracted from the chart and therefore not considered in the outcome.

The primary neonatal outcomes were small for gestational age (SGA, $<10$ th percentile) and large for gestational age (LGA, $>90$ th percentile). The PTB at $<37$ weeks was examined as a secondary neonatal outcome. Outcomes were selected due to the established increased risk for these maternal and neonatal events in women with chronic HTN.

Because superimposed preeclampsia is frequently associated with rapid weight gain due to edema, we assessed the timing of the GWG. The date of preeclampsia diagnosis was 
estimated from the date of admission to the hospital for delivery and/or the date of the diagnostic laboratory values, whichever was first. The patient's weight from the last prenatal visit prior to her preeclampsia diagnosis was used to estimate weight gain prior to preeclampsia, and GWG per week was calculated. To be included, the last weight measured prior to preeclampsia diagnosis had to be within 7 to 14 days prior to the diagnosis of preeclampsia. Among women who developed preeclampsia, weight gain in the weeks prior to the diagnosis of preeclampsia was examined separately from their weight gain for the pregnancy in its entirety.

Normal distribution of continuous variables was checked using the Kolmogorov-Smirnov test. The characteristics and outcomes of subjects gaining within, less than, or greater than the IOM recommendations were compared using analysis of variance (ANOVA) or chi-squared test for trend, as appropriate. Potentially confounding variables of the exposure-outcome association were identified in the stratified analysis. Multivariable logistic regression models for the outcomes of interest were then developed to estimate the effect of GWG above and below the IOM recommendations. Covariates were removed from the multivariable logistic regression models in a backward, stepwise fashion based on significant changes in the exposure adjusted odds ratio (AOR) or significant differences in the hierarchical models using likelihood ratio tests. Confounding factors considered included maternal age, parity, medication use prior to and during pregnancy, baseline renal disease, prior vaginal delivery, race, pregestational diabetes, and aspirin use. Because
BMI was used to classify women into their GWG category, it was not used in the multivariate models. All analyses were completed using Stata SE, version 13.1 (College Station, TX).

\section{Results}

Of 1,478 pregnancies complicated by chronic HTN identified over the study period, 776 were excluded (53 for major anomalies, 23 for no diagnosis of chronic HTN, 164 for major medical problems other than chronic HTN, diabetes or renal disease, 142 for no documented blood pressures prior to 20 weeks, 272 for incomplete BMI and GWG data, and 122 for being a subsequent pregnancy). Of the remaining 702 subjects, 106 (15.1\%) gained weight within IOM recommendations, 176 (25.0\%) gained weight less than IOM recommendations, and 420 (59.8\%) gained weight more than IOM recommendations. Maternal characteristics according to GWG group are shown in - Table 1. Women in each GWG category were similar with regard to maternal age, insurance type, history of pregnancy-induced HTN, prior cesarean delivery, medication use, and baseline renal disease (-Table 1). Women gaining weight greater than IOM recommendations were more likely to be nulliparous. Additionally, women gaining weight less than the IOM recommendations had a higher early pregnancy BMI compared with the within and more than the IOM recommendations groups.

The incidence of our primary maternal outcome, superimposed preeclampsia, increased across the GWG category (-Table 2). Similar increases in our secondary outcome of superimposed preeclampsia with severe features were also

Table 1 Maternal demographics

\begin{tabular}{|c|c|c|c|c|}
\hline & $\begin{array}{l}\text { Less than IOM } \\
\text { guidelines } \\
N=176\end{array}$ & $\begin{array}{l}\text { Within IOM } \\
\text { guidelines } \\
N=106\end{array}$ & $\begin{array}{l}\text { Above IOM } \\
\text { guidelines } \\
N=420\end{array}$ & p-Value \\
\hline Age & $30.8 \pm 5.4$ & $30.6 \pm 5.8$ & $29.9 \pm 6.1$ & 0.21 \\
\hline \multicolumn{5}{|l|}{ Race } \\
\hline $\begin{array}{l}\text { Black } \\
\text { White }\end{array}$ & $\begin{array}{l}144(81.8) \\
29(16.5)\end{array}$ & $\begin{array}{l}84(79.3) \\
19(17.9)\end{array}$ & $\begin{array}{l}285 \text { (67.9) } \\
108(25.7)\end{array}$ & $<0.01$ \\
\hline Hispanic & $1(0.6)$ & $1(0.6)$ & $17(4.1)$ & \\
\hline Other & $2(1.2)$ & $2(1.9)$ & $10(2.4)$ & \\
\hline Gov. insurance & $132(75.0)$ & $82(77.4)$ & $288(68.6)$ & 0.27 \\
\hline Smoking & $33(18.8)$ & $23(21.7)$ & $84(20.0)$ & 0.98 \\
\hline Nulliparity & $39(22.2)$ & $32(30.2)$ & $141(33.6)$ & 0.02 \\
\hline History of PIH & $54(30.7)$ & 38 (35.9) & $126(30.0)$ & 0.72 \\
\hline Anti-HTN medication (PTP) & $104(59.1)$ & $59(55.7)$ & $239(57.2)$ & 0.49 \\
\hline Anti-HTN medication in pregnancy & $138(78.4)$ & $88(83.0)$ & $329(78.3)$ & 0.55 \\
\hline$>1$ Anti-HTN medication required & $26(20.5)$ & $16(19.1)$ & $62(20.5)$ & 0.95 \\
\hline Early midtrimester BMI & $40.9 \pm 11.6$ & $40.3 \pm 11.6$ & $37.7 \pm 10.2$ & $<0.01$ \\
\hline Baseline renal disease & $15(8.5)$ & $7(6.6)$ & $45(10.7)$ & 0.38 \\
\hline
\end{tabular}

Abbreviations: BMI, body mass index; Gov, government; HTN, hypertension; IOM, Institute of Medicine; PIH, pregnancy-induced hypertension; PTP, prior to pregnancy.

Note: Unless otherwise specified, values are reported as the absolute number of patients with each outcome and the percentage in parentheses. 
e148 Gestational Weight Gain in Women with Chronic Hypertension Siegel et al.

Table 2 Maternal outcomes associated with gestational weight gain category

\begin{tabular}{|l|l|l|l|l|}
\hline & $\begin{array}{l}\text { Less than IOM guidelines } \\
N=176\end{array}$ & $\begin{array}{l}\text { Within IOM guidelines } \\
N=106\end{array}$ & $\begin{array}{l}\text { Above IOM guidelines } \\
N=420\end{array}$ & $p$-Value \\
\hline Superimposed preeclampsia & $25(14.2)$ & $25(23.6)$ & $123(29.3)$ & $<0.01$ \\
\hline Severe preeclampsia & $15(8.5)$ & $16(15.1)$ & $81(19.3)$ & $<0.01$ \\
\hline Cesarean delivery & $77(43.8)$ & $55(51.9)$ & $204(48.6)$ & 0.37 \\
\hline Primary cesarean & $45 / 136(33.1)$ & $26 / 76(34.2)$ & $119 / 309(38.5)$ & 0.50 \\
\hline
\end{tabular}

Abbreviation: IOM, Institute of Medicine.

Note: Data presented as N (\%).

observed across GWG category although no difference was observed in the mode of delivery. Additionally, statistically significant increases in LGA and PTB $<37$ weeks were observed across GWG category (-Table 3 ). The SGA was not different between the groups.

Results of the logistic regression analysis can be seen in -Table 4. After adjusting for significant confounding variables, weight gain less than the IOM recommendations compared with the weight gain within the IOM recommen- dations was associated with a lower risk of superimposed preeclampsia (AOR: 0.49, 95\% CI: 0.26-0.93) and superimposed preeclampsia with severe features (AOR: $0.44,95 \% \mathrm{CI}$ : 0.21-0.96) without an associated increase in the risk of SGA (AOR: 1.03, 95\% CI: 0.57-1.86).

Contrary to our hypothesis, weight gain above the IOM recommendations compared with weight gain within the IOM recommendations was not associated with an increased risk of superimposed preeclampsia (AOR: $1.20,95 \% \mathrm{CI}$ : $0.72-1.99$ ) or

Table 3 Neonatal outcomes associated with gestational weight gain category

\begin{tabular}{|l|l|l|l|l|}
\hline & $\begin{array}{l}\text { Less than IOM guidelines } \\
N=176\end{array}$ & $\begin{array}{l}\text { Within IOM guidelines } \\
N=106\end{array}$ & $\begin{array}{l}\text { Above IOM guidelines } \\
N=\mathbf{4 2 0}\end{array}$ & $p$-Value \\
\hline SGA & $36(20.5)$ & $22(21.0)$ & $62(14.8)$ & 0.13 \\
\hline LGA & $16(9.1)$ & $10(9.5)$ & $86(20.5)$ & $<0.01$ \\
\hline Birth weight $(\mathrm{kg})$ & $2,781 \pm 891$ & $2,873 \pm 847$ & $2,847 \pm 956$ & 0.65 \\
\hline Preterm birth $<37$ wks & $58(29.2)$ & $31(29.8)$ & $163(40.7)$ & 0.012 \\
\hline
\end{tabular}

Abbreviations: IOM, Institute of Medicine; LGA, large for gestational age; SGA, small for gestational age.

Note: Data presented as N (\%) or mean \pm standard deviation.

Table 4 Adjusted odds ratio for maternal and neonatal outcomes by multivariate logistic regression

\begin{tabular}{|c|c|c|}
\hline & Less than IOM guidelines & Above IOM guidelines \\
\hline \multicolumn{3}{|l|}{ Maternal } \\
\hline Superimposed preeclampsia ${ }^{a}$ & $0.49(0.26-0.93)$ & $1.20(0.72-1.99)$ \\
\hline Severe preeclampsia ${ }^{\mathrm{b}}$ & $0.44(0.21-0.96)$ & $1.20(0.66-2.19)$ \\
\hline Cesarean delivery $^{\mathrm{C}}$ & $0.65(0.38-1.10)$ & $0.76(0.49-1.19)$ \\
\hline Primary cesarean $^{\mathrm{d}}$ & $0.87(0.46-1.66)$ & $0.98(0.57-1.69)$ \\
\hline \multicolumn{3}{|l|}{ Neonatal } \\
\hline $\mathrm{SGA}^{\mathrm{e}}$ & $1.03(0.57-1.86)$ & $0.62(0.62-1.05)$ \\
\hline $\mathrm{LGA}^{\mathrm{e}}$ & $0.86(0.38-1.95)$ & $2.53(1.29-4.95)$ \\
\hline Preterm birth $<37$ wks $^{f}$ & $0.80(0.43-1.39)$ & $1.38(0.86-2.20)$ \\
\hline
\end{tabular}

Abbreviations: BMI, body mass index; IOM, Institute of Medicine.

Note: Reference group is within the IOM guidelines.

${ }^{a}$ Adjusted for medication use during pregnancy, baseline renal disease.

${ }^{\mathrm{b}}$ Adjusted for baseline renal disease.

'Adjusted for midtrimester BMI, prior vaginal delivery, pregestational diabetes.

${ }^{\mathrm{d} A d j u s t e d ~ f o r ~ m i d t r i m e s t e r ~ B M I, ~ p r i o r ~ v a g i n a l ~ d e l i v e r y, ~ m a t e r n a l ~ r a c e, ~ a n d ~ b a s e l i n e ~ r e n a l ~ d i s e a s e, ~ p r e g e s t a t i o n a l ~ d i a b e t e s . ~}$

${ }^{\mathrm{e}}$ Adjusted for midtrimester BMI.

${ }^{\mathrm{f}}$ Adjusted for baseline renal disease, prior preterm delivery, pregestational diabetes, and medication use during pregnancy. 
superimposed preeclampsia with severe features (AOR: 1.20, 95\% CI: 0.66-2.19). Additionally, weight gain above the recommendations was not associated with a significantly decreased risk of SGA (AOR: 0.62, 95\% CI: 0.62-1.05). Weight gain above the IOM recommendations was associated with a higher risk of LGA compared with weight gain within the IOM recommendations (AOR: 2.53, 95\% CI: 1.29-4.95).

In a secondary analysis, we assessed the timing of GWG compared with the diagnosis of superimposed preeclampsia. Of the 173 women diagnosed with superimposed preeclampsia, GWG data prior to their diagnosis of preeclampsia were available for 141 (81.5\%) women. Of the 18 women with superimposed preeclampsia and a total GWG below the IOM recommendations for the entire pregnancy, all 18 women had weight gain below the IOM recommendations in the 7 to 14 days prior to the preeclampsia diagnosis. Of the 23 women with preeclampsia and a total GWG within the IOM recommendations for the entire pregnancy, 19 (82.6\%) had weight gain within the IOM recommendations and 4 (17.4\%) had weight gain below the IOM recommendations 7 to 14 days prior to the preeclampsia diagnosis. In the 100 women who had weight gain above the IOM recommendations for the entire pregnancy, 92 (92.0\%) had weight gain greater than the IOM recommendations in the 7 to 14 days prior to preeclampsia diagnosis. Only eight women (8\%) who had weight gain greater than the IOM recommendations had it within the IOM recommendations prior to the preeclampsia diagnosis.

\section{Discussion}

In women with chronic HTN, weight gain greater than IOM recommendations was associated with an increased risk of LGA. We also found that GWG below IOM recommendations was associated with a decreased risk of superimposed preeclampsia without an associated increase in SGA. We found no difference in the mode of delivery for GWG above or below the IOM recommendations compared with weight gain within the IOM recommendations. Finally, we found that in women with chronic HTN ultimately diagnosed with superimposed preeclampsia, the weight gains largely happened prior to the onset of superimposed preeclampsia rather than due to edema associated with superimposed preeclampsia.

Previous studies have demonstrated a linear relationship between fetal growth, placental mass, and fetal weight. ${ }^{9}$ Furthermore, it has been shown that placental weight and size are reduced in women with hypertensive disorders of pregnancy and this is associated with unfavorable effects on fetal growth. ${ }^{10}$ Given the increased risk of placental insufficiency in this population and the association between GWG and birth weight, ${ }^{2}$ one may postulate that disproportionate GWG may be necessary in this group to overcome placental insufficiency and achieve an ideal birth weight. In contrast, we found that in our population, weight gain below IOM recommendations was associated with a decreased risk of superimposed preeclampsia without an associated increased risk of SGA.
Although similar research demonstrates a relationship between increased GWG and hypertensive disorders, previous studies have not examined subjects with chronic hypertension using IOM criteria. Jensen et al found an increased risk of hypertensive disorders with increased GWG in obese population. ${ }^{7}$ However, this study excluded women with chronic disease including HTN and also did not differentiate gestational HTN from preeclampsia. Additionally, Barton et al found that rates of both gestational HTN and preeclampsia increased with excessive weight gain in their cohort. ${ }^{11}$ In contrast to our study, they did not find an association between weight gain below recommendations and decreased incidence of gestational HTN and preeclampsia. This study, however, relied on data collected by telephonic interview and also did not complete a separate analysis for patients with a preexisting diagnosis of chronic HTN.

Madi et al investigated maternal and perinatal outcomes in women with a history of chronic HTN and found women with chronic HTN were more likely to have excessive weight gain (>16 kg), placental abruption, and higher rates of infants treated in the neonatal intensive care unit (NICU). ${ }^{12}$ This study also found an increased risk of cesarean delivery and SGA in their cohort with chronic HTN. However, this study did not perform a subgroup analysis using IOM criteria and evaluate for superimposed preeclampsia. ${ }^{12}$

We acknowledge that our study has several limitations. As a retrospective cohort study, we are inherently limited to observational data, and as such, we cannot assess the causes. This is essentially true of all studies of GWG, as patients cannot be randomized to a category. Also, we had fewer subjects in the less than and within GWG categories, and the majority of our cohort was overweight or obese. These characteristics of our cohort may have limited our ability to detect significant differences in rarer outcomes even though some of the trends are suggestive (and are areas to evaluate in more powerful studies). Additionally, although we analyzed women based on current IOM guidelines, the change in IOM guidelines during our study period may have resulted in subjects receiving slightly different weight gain counseling throughout the study period.

Our study also has several strengths, including the detailed chart abstraction of our cohort. Data were collected regarding maternal blood pressure, urine dipstick, and weight at each prenatal visit, maternal medication use, antepartum admissions, labor and delivery events, and neonatal outcomes. This enabled us to examine both total GWG as well as weight gain until a diagnosis of preeclampsia. It also enabled us to use current ACOG definitions of preeclampsia rather than relying on a charted or discharged diagnosis. Additionally, we classified subjects by GWG per week for BMI category instead of weight gain as a whole. Given that longer gestations will inherently have GWG, a consideration of it per week may help to control for length of gestation, an important factor in this type of study.

To our knowledge, this is the first large cohort study examining the IOM recommendations for GWG in women with chronic HTN. A search of the PubMed database using 
search terms "gestational weight gain" and "chronic hypertension" did not yield any reports focusing on women with chronic HTN to address this question. In this cohort, increasing GWG was associated with an increased risk of LGA infants while gestational weight below IOM recommendations was associated with a decreased risk of superimposed preeclampsia without an increased risk of SGA. Therefore, women with chronic HTN should be counseled to gain no more than the IOM recommendations. Further research is required to confirm that women with chronic HTN should have lower weight gain recommendations than current targets.

\section{Conflict of Interests}

The authors report no conflict of interests.

Note

The findings were presented at the 36th Annual Society of Maternal Fetal Medicine Pregnancy meeting in Atlanta, GA from February 2 to 6, 2016 at a poster session.

\section{Acknowledgments}

Dr. Harper is supported by K12HD001258. We are grateful to PI Andrews, who partially supported this work.

\section{References}

1 Sibai BM. Chronic hypertension in pregnancy. Obstet Gynecol 2002;100(02):369-377

2 Institute of Medicine. Weight Gain During Pregnancy: Reexamining the Guidelines. Washington, DC: The National Academies Press; 2009
3 Liu J, Wang XF, Wang Y, Wang HW, Liu Y. The incidence rate, highrisk factors, and short- and long-term adverse outcomes of fetal growth restriction: a report from Mainland China. Medicine (Baltimore) 2014;93(27):e210

4 Veerbeek JH, Nikkels PG, Torrance HL, et al. Placental pathology in early intrauterine growth restriction associated with maternal hypertension. Placenta 2014;35(09):696-701

5 Chandrasekaren S, Levine LD, Durnwald CP et al. Excessive weight gain and hypertensive disorders of pregnancy in the obese patient. J Matern Fetal Neonatal Med 2015;28(08):964-968

6 Thorsdottir I, Torfadottir JE, Birgisdottir BE, Geirsson RT. Weight gain in women of normal weight before pregnancy: complications in pregnancy or delivery and birth outcome. Obstet Gynecol 2002; 99(5, Pt 1):799-806

7 Jensen DM, Ovesen P, Beck-Nielsen H, et al. Gestational weight gain and pregnancy outcomes in 481 obese glucose-tolerant women. Diabetes Care 2005;28(09):2118-2122

8 The American College of Obstetrics and Gynecologists. Optimizing Protocols in Obstetrics. Key Elements for the Management of Hypertensive Crisis in Pregnancy (In-patient). Series 4. 2013. Available at http://www.acog.org/-/media/Districts/District-II/ MembersOnly/PDFs/Optimizing-Protocols-In-OB-HTN-Series-3Version1.pdf

9 Molteni RA, Stys SJ, Battaglia FC. Relationship of fetal and placental weight in human beings: fetal/placental weight ratios at various gestational ages and birth weight distributions. J Reprod Med 1978;21(05):327-334

10 Salmani D, Purushothaman S, Somashekara SC, et al. Study of structural changes in placenta in pregnancy-induced hypertension. J Nat Sci Biol Med 2014;5(02):352-355

11 Barton JR, Joy SD, Rhea DJ, Sibai AJ, Sibai BM. The influence of gestational weight gain on the development of gestational hypertension in obese women. Am J Perinatol 2015;32(07):615-620

12 Madi JM, Araújo BF, Zatti H, et al. Chronic hypertension and pregnancy at a tertiary-care and university hospital. Hypertens Pregnancy 2012;31(03):350-356 